\title{
De Novo Posttransplant Thrombotic Microangiopathy Localized Only to the Graft in APKD with Thrombophilia
} \author{
and Matteo Trezzi ${ }^{1}$ \\ ${ }^{1}$ Divisione di Nefrologia - Dialisi - Trapianto, Ospedale Sant'Andrea, La Spezia, Italy \\ ${ }^{2}$ Divisione di Chirurgia Vascolare e Trapianto di rene, Azienda Ospedaliera Universitaria San Martino, Genova, Italy \\ ${ }^{3}$ Servizio di Anatomia Patologica, Azienda Ospedaliera Universitaria San Martino, Genova, Italy \\ ${ }^{4}$ Clinica Nefrologica, Azienda Ospedaliera Universitaria San Martino, Genova, Italy \\ ${ }^{5}$ Estor Spa, Pero, Italy
}

Davide Rolla ${ }^{\star}$, Iris Fontana ${ }^{2}$, Jean Louis Ravetti ${ }^{3}$, Luigina Marsano ${ }^{4}$, Diego Bellino ${ }^{4}$, Laura Panaro ${ }^{4}$, Francesca Ansaldo ${ }^{4}$, Lisa Mathiasen ${ }^{5}$

\begin{abstract}
Background: Thrombotic microangiopathy (TMA) is a serious complication of renal transplantation (Tx) and is mostly related to the prothrombotic effect of calcineurin inhibitors (CNIs). A subset of TMA (29-38\%) is localized only to the graft.

Case 1: A young woman suffering from adult polycistyc kidney disease (APKD) underwent kidney transplant. After 2 months, she showed slow renal deterioration (Scr from 1.9 to $3.1 \mathrm{mg} / \mathrm{dl}$ ), without hematological signs (anaemia, low platelets, low serum haptoglobins, schistocytosis $>1 \%$ ) of haemolytic-uremic syndrome (HUS); only lactate dehydrogenase enzyme (LDH) transient increase was detected. Renal biopsy showed TMA: temporary withdraw of tacrolimus and plasmapheresis was performed. The renal function recovered and stabilized (Scr $1.9 \mathrm{mg} / \mathrm{dL}$ ). From Screening for thrombophilia, we found a mutation of the Leiden Factor $\mathrm{V}$ gene.

Case 2: An old man affected by APKD underwent Tx, with delayed graft function (DGF); first biopsy showed acute tubular necrosis, but a second biopsy on day 45 revealed TMA; no altered hematological parameters of HUS was detected. Again, we observed only a light increase of LDH levels. The tacrolimus was halved and plasmapheresis was performed: LDH levels normalized within 10 days and renal function improved (Scr from 9 to $2.9 \mathrm{mg} / \mathrm{dl}$ ). In this patient, we found a mutation of the Prothrombin gene.

Only a renal biopsy clarifies the diagnosis of TMA localized into the graft, but it is necessary to pay attention to light increasing level of LDH.

Conclusions: Prothrombotic effect of $\mathrm{CNIs}$ and $\mathrm{M}$-Thor inhibitor, mutation of genes encoding factor $\mathrm{H}$ or $\mathrm{I}$, anticardiolipin antibodies, vascular rejection, cytomegalovirus or parvovirus B19 infection are proposed to trigger TMA we detected mutations of Factor II and Leiden Factor V, as facilitating conditions for TMA in patients affected by APKD.
\end{abstract}

Keywords: Thrombotic miocroangiopathy; Kidney transplant; Leiden factor V; Protrombin gene

\section{Introduction}

Thrombotic microangiopathy (TMA) is a pathological process of microvascular thrombosis, thrombocitopenia and microangiopathic haemolytic anaemia, with ischaemia and occasionally infarction, affecting particularly the kidney and the brain [1]. TMA is a recognized and devastanting process in renal transplantation, affecting 3\% to $14 \%$ of patients on calcineurin inhibitors (CNIs) therapy [2-5]. It produces narrowing or occlusion of capillaries, fibrinoid change in the intima of small arteries and subendothelial accumulation of amorphous material in glomeruli. A subset of TMA (29-38\%) is localized only to the graft and patients do not show the classical signs of hemolytic uremic syndrome.

The clinical presentation of TMA is variable, often shows systemical signs of haemolytic uraemic syndrome (HUS), with findings of haemolytic-anemia, rapid deterioration of renal function, peripheral schistocytes, and thrombocytopenia, but cases of TMA only localized to the allograft do not present systemic manifestation of HUS.

CNIs, mTOR inhibitors, viral infections, ischemia-reperfusion injury, acute rejection, mutation of genes encoding factor $\mathrm{H}$ or $\mathrm{I}$, anticardiolipin antibodies predispose to post-transplant TMA [6,7], but no coagulation genetic alterations were described to facilitate this serious complication of renal transplantation.
We describe two cases of de novo TMA localized only to the graft in adult polycystic kidney disease (APKD) with thrombophilia.

\section{Case I}

A 39-year-old-woman, suffering from APKD, diagnosed at 14-yearsold owing to gross haematuria, was on hemodialysis for one year before the transplant. The patient received a kidney transplant (Ktx) from a 52 years-old deceased due to cerebral hemorrage with 14 hours of cold ischemic time. The HLA mismatch was 1 and his immunosuppression consisted of two intravenous doses of basiliximab, the first dose (20 $\mathrm{mg}$ ) preoperatively and the second dose $(20 \mathrm{mg})$ on postoperative day 4 , a methyprednisolon taper to $5 \mathrm{mg}$ daily, mycophenolate and tacrolimus as maintenance. We observed an incomplete recovery of

*Corresponding author: Davide Rolla, Divisione di Nefrologia-Dialisi-Trapianto, Osp. Sant'Andrea, La Spezia, Italy, Tel: +39 0187533213; Fax: +390187533045 E-mail: davide.rolla@asl5.liguria.it

Received September 18, 2015; Accepted September 30, 2015; Published October 08, 2015

Citation: Rolla D, Fontana I,Ravetti JL, Marsano L, Bellino D, et al. (2015) De Novo Posttransplant Thrombotic Microangiopathy Localized Only to the Graft in APKD with Thrombophilia. J Kidney 1: 101. doi:10.4172/2472-1220.1000101

Copyright: @ 2015 Rolla D, et al. This is an open-access article distributed under the terms of the Creative Commons Attribution License, which permits unrestricted use, distribution, and reproduction in any medium, provided the original author and source are credited. 
Citation: Rolla D, Fontana I,Ravetti JL, Marsano L, Bellino D, et al. (2015) De Novo Posttransplant Thrombotic Microangiopathy Localized Only to the Graft in APKD with Thrombophilia. J Kidney 1: 101. doi:10.4172/2472-1220.1000101

renal function (Scr $1.9 \mathrm{mg} / \mathrm{dL}$ ). After 2 months from Ktx, she showed a slow renal deterioration (until Scr $3.1 \mathrm{mg} / \mathrm{dL}$ ), without humoral signs of hemolytic syndrome; only a transient increase of lactate dehydrogenase enzyme (LDH) was seen (Figure 1). An allograft kidney biopsy was performed and renal biopsy specimen showed fragmented red blood cells in the glomeruli, partial occlusion of capillaries, fibrinoid change in the intima of small arteries and subendothelial accumulation of amorphous material (Figures 2-4). Immediately, temporary withdraw of TAC was performed and a plasmapheresis cycle (five sessions of plasma-exchanges) was made. The renal function slowly recovered and it was stabilized (Scr $1.9 \mathrm{mg} / \mathrm{dl}$ ) after two years from the de novo TMA. Genetic coagulation abnormality study was performed and we found an heterozygous mutation of Factor V Leiden gene. Search for mutations of gene encoding factor $\mathrm{H}$ and I was negative.

\section{Case II}

In a 68-year-old man, with the mother affected by polycystic kidney disease, suffering from arterial hypertension and previous sustained tabagism, APKD was diagnosed at 57-years-old. In 2012, he underwent Ktx from a 60 years-old woman deceased due cerebral hemorrhage, with 15 hours of cold ischemic time. The HLA mismatch was 4 and the immunosuppression consisted of two intravenous doses of basiliximab (20 $\mathrm{mg}$ ), a methyprednisolon taper to $5 \mathrm{mg}$ daily, mycophenolate and tacrolimus as maintenance. A prolonged DGF with oligo-anuria characterized the clinical course; a first biopsy after 10 days showed NTA, a second biopsy does not contain glomeruli, and a third biopsy, done at day 45 showed a severe TMA. The renal biopsy specimen demonstrated arteriolar thrombi, occlusion of glomerular capillaries by amorphous material, fragmented red blood cells in glomeruli (Figures 5-7). No signs of haemolytic uremic syndrome were detected. Again, we observed only a persistent slight increase of LDH. The TAC was halved and LDH levels, in addition to renal function, were strictly observed: lactate dehydrogenase enzyme levels normalized within 10 days, diuresis increased and became effective with partial, but satisfactory recovery of renal function (Scr $2.9 \mathrm{mg} / \mathrm{dL})$. After four years from the de novo TMA, we observed further amelioration ( $\mathrm{Scr}$ $1.5 \mathrm{mg} / \mathrm{dL}$ ) Also for this patient, genetic coagulation abnormality study was performed, and we found an heterozygous mutation of the Prothrombin gene (Factor II).

\section{Discussion}

Thrombotic microangiopathy is a serious complication of renal

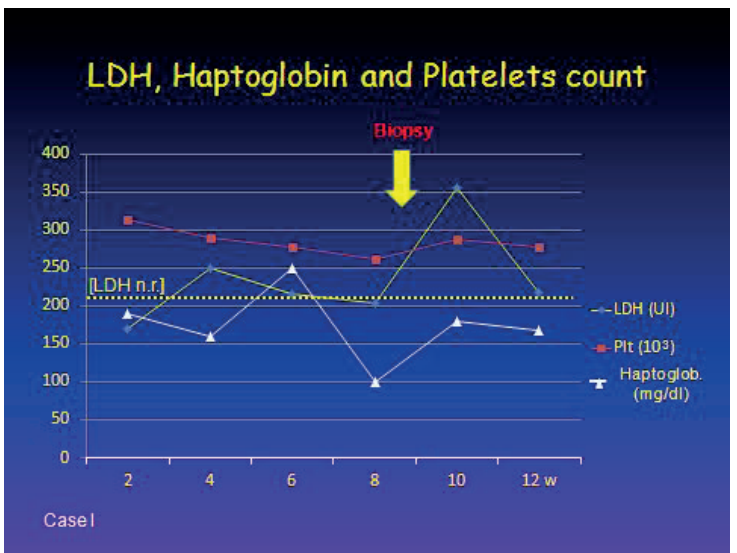

Figure 1: Case I. Lactate dehydrogenase enzyme (LDH) value, haptoglobin level and platelets count before and after biopsy, that showed localized thrombotic microangiopathy after two months from kidney transplant.

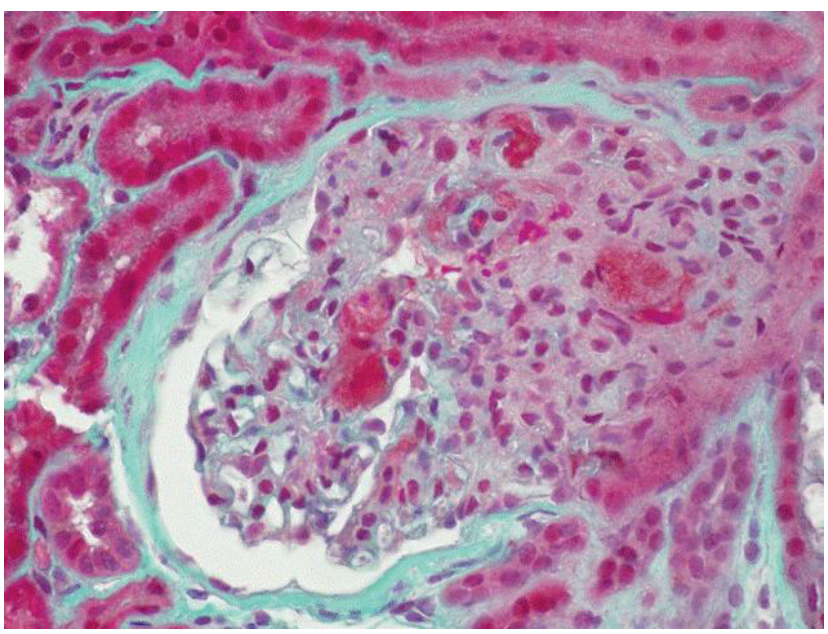

Figure 2: Case I. Renal biopsy specimen demonstrated histopathological glomerular characteristics of TMA (400x). Dilatation of capillaries, stasis and thrombosis (Masson's trichrome).

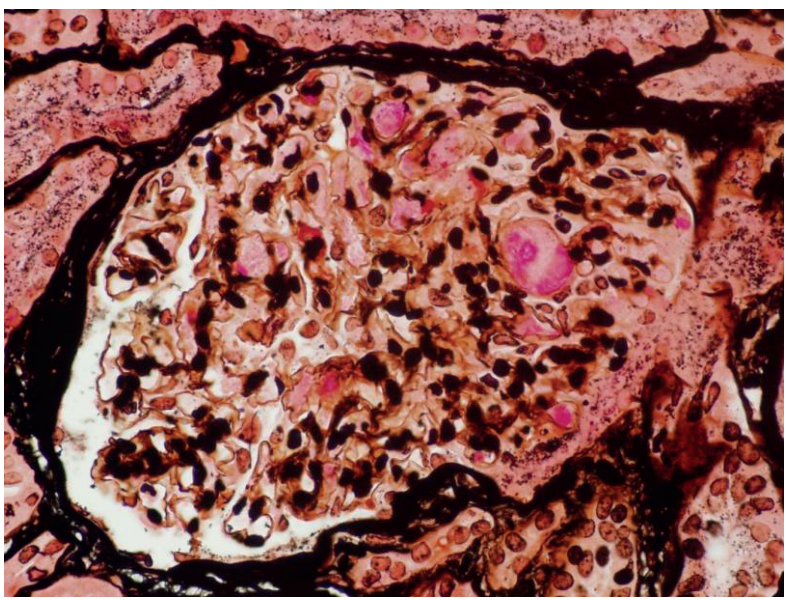

Figure 3: Case I. Renal biopsy specimen demonstrated histopathological glomerular characteristics of TMA (400x). Thrombosis in dilated capillary lumina (Periodic Schiff-Methenamine).

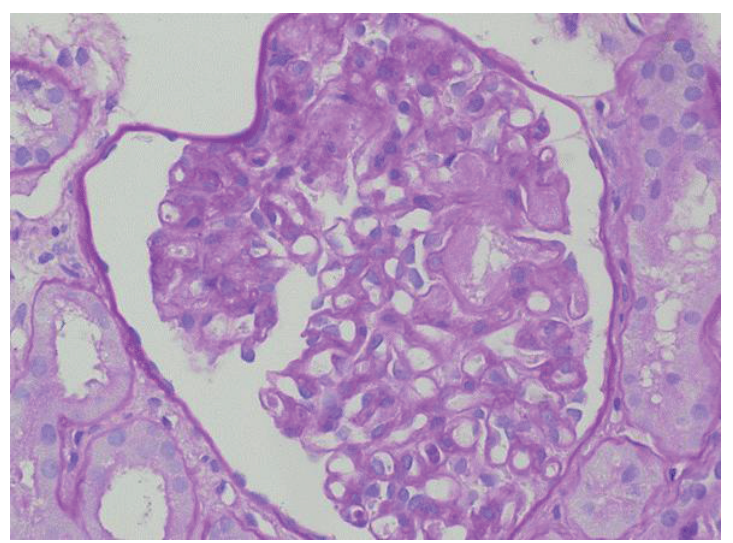

Figure 4: Case I. Renal biopsy specimen demonstrated histopathological glomerular characteristics of TMA (400x). Mesangiolysis and dilated capillary lumina (Periodic Acid Schiff). 
Citation: Rolla D, Fontana I,Ravetti JL, Marsano L, Bellino D, et al. (2015) De Novo Posttransplant Thrombotic Microangiopathy Localized Only to the Graft in APKD with Thrombophilia. J Kidney 1: 101. doi:10.4172/2472-1220.1000101

Page 3 of 4

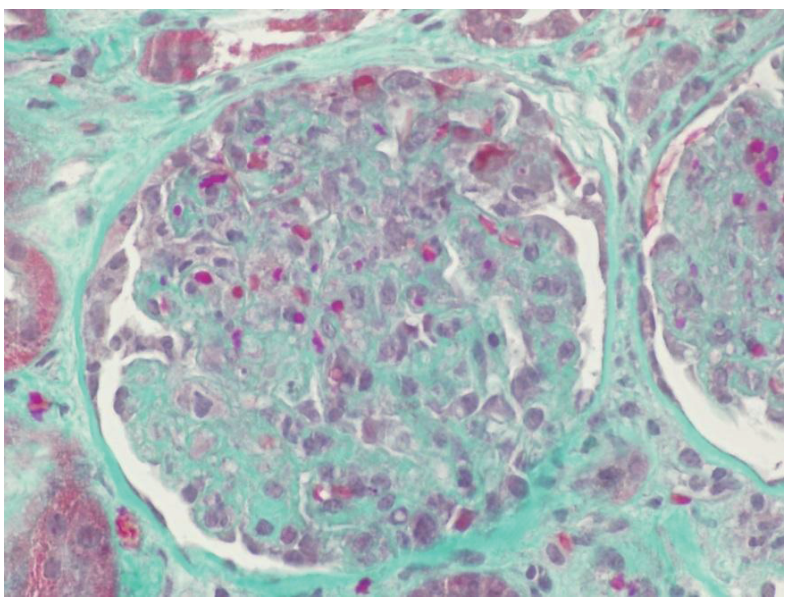

Figure 5: Case II. Renal biopsy specimen showed glomerular lesions in TMA (400x). Focal fibrinoid necrosis. (Masson's trichrome).

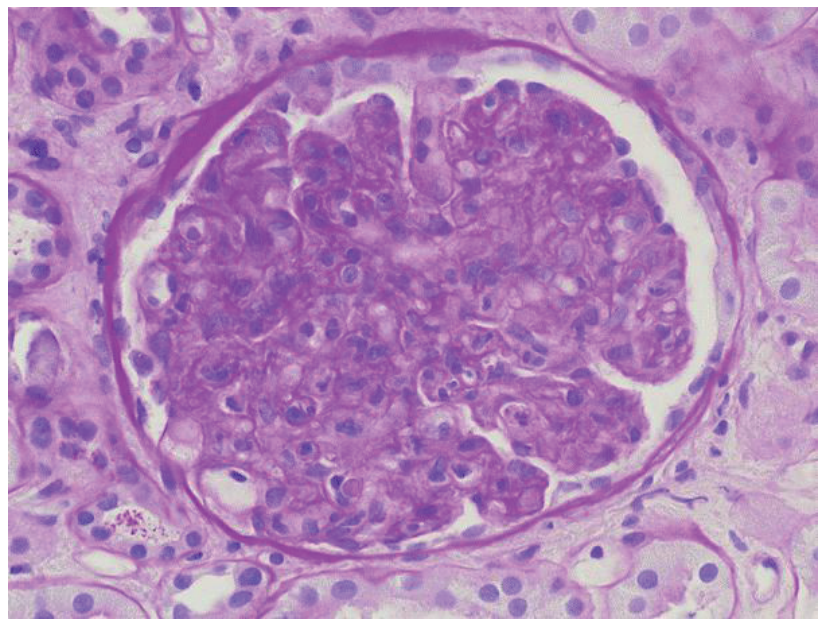

Figure 6: Case II. Renal biopsy specimen showed glomerular lesions in TMA (400x). Focal fibrinoid necrosis. (Masson's trichrome). Most capillary lumina closed. (PAS).

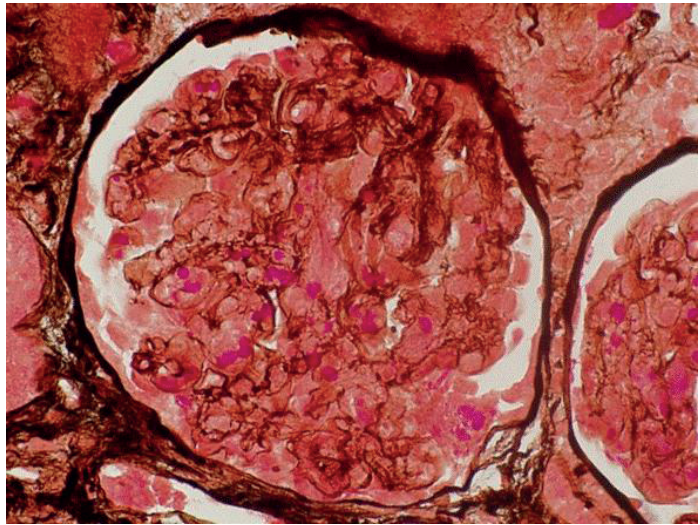

Figure 7: Case II. Renal biopsy specimen showed glomerular lesions in TMA (400x). Focal fibrinoid necrosis. (Masson's trichrome). Thickening of the capillary walls and thrombosis (PASM). transplantation, affecting $3 \%$ to $14 \%$ of patients. It can occur as a recurrent or de novo disease. In a retrospective review by Schwimmer J et al (2) between 21 of 742 kidney transplant recipients suffering from TMA, 38\% showed localized thrombotic microangiopathy. Patients with systemic and localized TMA did not differ significantly with respect to age, sex, race, type of donor and pre-transplant diagnosis, and despite systemic and localized TMA have different characteristics and clinical course, long-term graft survival was similarly poor (graft loss 14 to $57 \%$ in published case series). Conversely, in other published studies $[8,9]$, a high proportion of patients suffering from localized TMA showed lower rates of TMA-related graft loss (0 to 12\%).

We have described two cases of kidney transplant suffering from TMA due CNIs, facilitated by genetic coagulation abnormalities, with a favourable outcome and a very good follow-up.

Toxicity by CNIs is the main etiologic factor of the disease, because CNIs induce arteriolar vasoconstriction due to production of vasoconstrictive factors, for increased sensitivity to endothelin-I and low synthesis of prostacyclin or nitric oxide. Furthermore, CNIs could promote a procoagulant state by enhancing platelet aggregation [10].

Moreover, mTor inhibitors, viral infection, ischemia-reperfusion injury, viral infection (CMV-Parvovirus B19 and Polioma BK) antibody-mediated rejection and antiphospholipides antibodies are other frequent etiologic agents.

The coagulation abnormality is not investigated enough in this disease, although microvascular thrombosis is the hallmark of TMA.

We have described two different anomalies of coagulation state in APKD patients. The mutation of Factor V Leiden (three heterozygous, and one homozygous) was described by Raife [11] in a white TMA patient, who had normal von Willebrand factor-cleaving protease (VWCP). On the other hand, no factor II allele abnormality was detected in patients suffering from TMA with normal or deficient VWCP.

Inactivation of Factor $\mathrm{Va}$ by activated protein $\mathrm{C}$ is impaired in patients with Factor Leiden mutation. It's possible that it is implicated in the thrombomodulin/protein $\mathrm{C}$ anticoagulant pathway in TMA.

In conclusion, Factor $\mathrm{V}$ Leiden may be among a constellation of genetic risks permissive of the development of TMA, when CNIs exposition is present, as in the cases we have described here.

Localized TMA has a variable clinical presentation, as no signs of haemolytic anaemia, schistocytes, thrombocytopenia and low aptoglobin level were observed. Only a moderate increase of lactate dehydrogenase enzyme was seen and only biopsy is really diagnostic.

\section{References}

1. Moake JL (2002) Thrombotic microangiopathies. N Engl J Med 347: 589-600.

2. Schwimmer J, Nadasdy TA, Spitalnik PF, Kaplan KL, Zand MS (2003) De novo thrombotic microangiopathy in renal transplant recipients: a comparison of hemolytic uremic syndrome with localized renal thrombotic microangiopathy. Am J Kidney Dis 41: 471-479.

3. Reynolds JC, Agodoa LY, Yuan CM, Abbott KC (2003) Thrombotic microangiopathy after renal transplantation in the United States. Am J Kidney Dis 42: $1058-1068$.

4. Ardalan MR (2006) Review of thrombotic microangiopathy (TMA), and postrenal transplant TMA. Saudi J Kidney Dis Transpl 17: 235-244.

5. Nava F, Cappelli G, Mori G, Granito M, Magnoni G, et al. (2014) Everolimus, cyclosporine, and thrombotic microangiopathy: clinical role and preventive tools in renal transplantation. Transplant Proc 46: 2263-2268. 
Citation: Rolla D, Fontana I,Ravetti JL, Marsano L, Bellino D, et al. (2015) De Novo Posttransplant Thrombotic Microangiopathy Localized Only to the Graft in APKD with Thrombophilia. J Kidney 1: 101. doi:10.4172/2472-1220.1000101

Page 4 of 4

6. Noris M, Remuzzi G (2010) Thrombotic microangiopathy after kidney transplantation. Am J Transplant 10: 1517-1523.

7. Barbour T, Johnson S, Cohney S, Hughes P (2012) Thrombotic microangiopathy and associated renal disorders. Nephrol Dial Transplant 27: 2673-2685.

8. Zarifian A, Meleg-Smith S, O'donovan R, Tesi RJ, Batuman V (1999) Cyclosporine-associated thrombotic microangiopathy in renal allografts. Kidney Int 55: 2457-2466

9. Young BA, Marsh CL, Alpers CE, Davis CL (1996) Cyclosporine-associated thrombotic microangiopathy/hemolytic uremic syndrome following kidney and kidney-pancreas transplantation. Am J Kidney Dis 1996 28: 561-571.

10. Caires RA, Marques ID, Repizo LP, Sato VA, Carmo LP, et al. (2012) De novo thrombotic microangiopathy after kidney transplantation: clinical features, treatment, and long-term patient and graft survival. Transplant Proc 44: 2388 2390.

11. Raife TJ, Lentz SR, Atkinson BS, Vesely SK, Hessner MJ (2002) Factor V Leiden: a genetic risk factor for thrombotic microangiopathy in patients with normal von Willebrand factor-cleaving protease activity. Blood 99: 437-442. 